\title{
The effect of presentation level on memory performance
}

\section{Document Version}

Final published version

Link to publication record in Manchester Research Explorer

\section{Citation for published version (APA):}

Heinrich, A., \& Schneider, B. A. (2011). The effect of presentation level on memory performance. Ear and hearing, $32(4), 524-532$.

\section{Published in:}

Ear and hearing

\section{Citing this paper}

Please note that where the full-text provided on Manchester Research Explorer is the Author Accepted Manuscript or Proof version this may differ from the final Published version. If citing, it is advised that you check and use the publisher's definitive version.

\section{General rights}

Copyright and moral rights for the publications made accessible in the Research Explorer are retained by the authors and/or other copyright owners and it is a condition of accessing publications that users recognise and abide by the legal requirements associated with these rights.

\section{Takedown policy}

If you believe that this document breaches copyright please refer to the University of Manchester's Takedown Procedures [http://man.ac.uk/04Y6Bo] or contact uml.scholarlycommunications@manchester.ac.uk providing relevant details, so we can investigate your claim.

\section{OPEN ACCESS}




\title{
The Effect of Presentation Level on Memory Performance
}

\author{
Antje Heinrich and Bruce A. Schneider
}

\begin{abstract}
Objectives: A loss of speech intelligibility at high presentation levels is called rollover. It is a phenomenon that increases in prevalence as people age. Whether the adverse effect of high presentation levels extends to processes subsequent to speech intelligibility, such as memory, is unknown. The present study examined this question on the basis of the previous finding that older but not younger adults showed memory impairment when acoustically distorted words were presented at $50 \mathrm{~dB}$ SL compared with an undistorted baseline presented at $65 \mathrm{~dB}$ $\mathrm{SPL}$. One question investigated in the present study was whether a presentation level of $50 \mathrm{~dB}$ SL put older listeners at the cusp of rollover and whether this subsequently impaired memory. Moreover, we wanted to know whether and at what level it was possible to induce a similar impairment in younger listeners.
\end{abstract}

Design: We used a paired-associate memory paradigm in which five word pairs per list were presented at a rate of 4 secs per word pair. After each list, the first word of one of the pairs was presented again and the listener was asked to recall the second word. Over the course of the experiment, all list positions were tested an equal number of times. The word pairs, which were acoustically distorted using a jittering algorithm, were presented at $40 \mathrm{~dB}$ SL to all younger and older participants and just below an uncomfortably loud level for younger listeners only. Intelligibility of the distorted words was equated across age groups for each presentation level. The effect of presentation level on memory performance was investigated and compared with data of a previous study that used the same design but presented the distorted and undistorted words at $50 \mathrm{~dB} S \mathrm{SL}$ to both age groups. A total of 58 younger and 24 older adults were tested in two experiments.

Results: The results showed that for older adults, memory performance for distorted words was decreased in all list positions at a presentation level of $50 \mathrm{~dB}$ SL compared with $40 \mathrm{~dB}$ SL and an undistorted $65 \mathrm{~dB}$ SPL baseline. This effect did not occur for younger listeners. However, when younger adults were tested at a very high presentation level, they showed the same memory decrease compared with the baseline as older adults showed for $50 \mathrm{~dB}$ SL.

Conclusions: A high presentation level of distorted words can adversely affect memory even after intelligibility is equated for. Moreover, older listeners are affected at lower presentation levels. Hence, the choice of sound level, particularly for older listeners, is important and may affect their level of cognitive performance beyond its effects on intelligibility. Higher presentation levels may not always lead to better performance when the task involves recall of words previously heard.

(Ear \& Hearing 2011;32;524-532)

\section{INTRODUCTION}

Audiologists and hearing scientists are beginning to recognize that the implications of hearing loss go beyond speech recognition and include things such as comprehension, inference making, and memory (Pichora-Fuller \& Singh 2006). In fact, the ability to store and recall heard information is an important prerequisite for speech compre-

Department of Psychology, University of Toronto at Mississauga, Mississauga, Ontario, Canada. hension. However, the relationship between successful word perception and successful subsequent memory encoding is not as straightforward as one might think. Several studies have found that under some circumstances, noisy backgrounds can impair a listeners' ability to recall words previously heard, even when their ability to recognize the words, when they are spoken, is at or near ceiling (Rabbitt 1968; Pichora-Fuller et al. 1995; Murphy et al. 2000; Heinrich et al. 2008; Sarampalis et al. 2009; Heinrich \& Schneider 2010). Although the circumstances in which words that are correctly perceived but are not adequately encoded in memory are not fully understood, the results to date suggest that the effect is more severe in older adults and those with hearing loss. Both of these groups tend to suffer from reduced audibility and are, as a consequence, subjected to higher presentation levels, either through talking louder or by amplifying the sound. However, we know that the benefit of raising the presentation level to compensate for sensitivity loss is limited and that when presentation levels become too high, the result is a reduction rather than an increase in intelligibility (the rollover effect; Jerger \& Jerger 1971; Dirks et al. 1977; Bess et al. 1979). This raises the possibility that a similar limitation to raised presentation levels exists for memory and that a listening situation can exist in which words, although still correctly recognized, become difficult to encode into memory if the presentation level is too high. In this study, we examine the extent to which the introduction of distortion affects memory and how memory for distorted words is affected by presentation level.

\section{Memory in Noisy Backgrounds}

A number of studies have examined the mechanisms by which noise interferes with memory and recall (Pichora-Fuller et al. 1995; Murphy et al. 2000; Schneider et al. 2000; Heinrich et al. 2008; Heinrich \& Schneider 2010). For instance, in a series of experiments, Murphy et al. (2000) and Heinrich et al. $(2008,2010)$ used a paired-associate memory paradigm that was modeled based on that used in the study by Madigan and McCabe (1971). In this paradigm, lists of five word pairs were presented to younger and older listeners at a rate of 4 secs per word pair in quiet and in various babble (12 people talking simultaneously) backgrounds. Eight seconds after the presentation of the last pair, the participant was presented with the first word of one of the pairs and asked to provide its companion word. In this paradigm, the term "serial position" refers to the presented word pairs such that the first serial position refers to the first word pair and so forth. It was assumed that the last two pairs of a list depended on working memory for recall, whereas the more temporally remote first three word pairs depended on a longer-term episodic memory 
system for successful encoding and retrieval (Madigan \& McCabe 1971; Craik \& Lockhart 1972; Floden et al. 2000).*

In a first step, Murphy et al. (2000) presented the lists of word pairs to younger and older adults in a quiet background and in a background of continuous 12-talker babble. In quiet, younger and older adults performed equivalently in serial positions 4 and 5, but younger adults recalled more words than older adults in serial positions 1 to 3 . In a continuous background babble that was adjusted to provide comparable intelligibility between the two age groups, younger adults' memory performance in positions 1 to 3 looked very similar to that of older listeners in quiet. However, older adults' memory performance was further decreased in all serial positions in a continuous babble background compared with quiet even though the intelligibility of the words in babble was equated to match that of younger listeners. Hence, noise can adversely affect memory in both younger and older adults, with the memory effects being larger in older than in younger adults.

These results are consistent with the notion that the attentional resources that individuals can bring to bear on a problem are limited (Kahneman 1973; Craik 2002). When listening in noise, people have to divide their attentional resources between the task of extracting the words from the babble background and placing them in working memory and the task of transferring them from working memory to episodic memory (for pairs 1 to 3). To extract the signal, listeners first have to segregate the noise from the target (stream segregation; Bregman 1990), a process that has been shown to require attention when complex sounds are involved (Snyder \& Alain 2005; Alain \& McDonald 2007). Transferring information from working memory to a more stable and long-lasting (Craik \& Lockhart 1972; Craik 1983; Floden et al. 2000) episodic memory also requires attentional resources (Craik 2002). If resources are limited, either because of older age or because part of the same resources are involved in segregating the target words from babble background, recall from the first three serial positions can be expected to suffer first. Hence, the reason why older adults tested in quiet and younger adults tested in babble performed similarly in the study by Murphy et al. could reflect resource limitations in both instances. In older adults, the resources required for transferring words into episodic memory may be limited because of age-related changes in cognitive processes. In younger adults, this resource limitation may be a consequence of splitting attentional resources between stream segregation and transfer of words into episodic memory. The poorer performance of older adults in a babble background in all serial positions suggests that the combination of age and noise places such a strain on attentional resources that both episodic and working memory are affected in older adults.

In follow-up experiments (Heinrich et al. 2008; Heinrich \& Schneider 2010) designed to characterize the influence of background babble on the episodic memory performance in more detail, it was shown that presenting the babble back-

\footnotetext{
*Working memory is responsible for the processing and temporary storage of task-relevant information during the performance of everyday cognitive tasks such as speech (Carpenter \& Just 1989). Here, we assume that the last two presented words are still available and retrievable from working memory. Episodic memory refers to the long-term storage of past events or episodes (Hebb 1949; Brown 1958; Peterson \& Peterson 1959). It is assumed here that words from the more remote serial positions have been transferred from working memory to episodic memory and are retrieved from there.
}

ground only between word pairs or gated on and off with the word pairs resulted in performance in the first three serial positions that was equivalent to that found in quiet for both younger and older adults. However, when babble was presented 500 msecs before the onset of a word pair and continued until 500 msecs after the offset of the last word in the pair, it produced the same decrement in the early serial positions in both age groups as that produced by continuous babble. These results indicated that babble only has an adverse effect on episodic memory in younger and older adults when it precedes and follows (flanks) a pair. Presumably, when the babble precedes the word pairs (or is on continuously), attentional resources that otherwise could be used to transfer the word pairs from working memory to episodic memory are diverted to stream segregation to better facilitate the extraction of the target words from the noise, thereby reducing the time that they are available to episodic memory processes.

It is interesting to note that the effects of babble during word presentation had a differential effect on working memory (serial positions 4 and 5) in younger and older adults. For younger adults, recall in positions 4 and 5 was unaffected by continuous, flanked, or gated babble. However, the recall performance of older adults in positions 4 and 5 was reduced by an equivalent amount for continuous and flanked babble, with the reduction being even more severe when the babble was gated on and off with the word pairs. One interpretation of this age effect is that stream segregation is slower in older than in younger adults. Slower stream segregation in older adults would lead to slower and less efficient lexical access, thereby affecting working memory operations, and, consequently, recall in positions 4 and 5. Moreover, we would expect the effect of babble on working memory to be less severe (as it was) when the babble is on before the words are presented, because the older adult has more time to build up the perception of a background babble before the target words are presented. The lack of an effect of babble on working memory in younger adults suggests that stream segregation is more rapid for them than it is for older adults.

Another possible explanation for older adult's poorer performance when the babble was gated on with the word pairs is that the simultaneous onset of the babble and word pairs required the immediate reallocation of attentional resources to permit listeners to overcome the phonemic degradation caused by the babble (Warren 1970; Ronnberg et al. 2008). If older adults were either slower to deploy or had fewer attentional resources than younger adults to devote to phonemic recovery, or if phomemic recovery required more of these resources in older than in younger adults, older adults would have fewer attentional resources available to them for memory processes. Hence, either slower stream segregation or a greater need for and perhaps slower deployment of attentional resources to recover from phonemic distortion would result in older adults' memory being more vulnerable in a noisy background.

Further evidence that working memory (recall performance in serial positions 4 and 5) is more susceptible to interference in older adults than in younger adults comes from studies in which the word pairs, rather than being masked by noise, are distorted instead (Heinrich \& Schneider 2010). When word pairs were degraded by physically distorting (jittering) them rather than presenting them in babble, older listeners also showed a decrease for the last two word pairs under one of the 
two jitter conditions, whereas younger adults again remained unaffected throughout. (Note that in the spectral domain, distorting the words is equivalent to presenting the words in a band of noise that is gated on and off with word presentation.) Hence, the poorer performance for distorted words by older adults in the last two serial positions could reflect a slowing of access to the lexicon due to the introduction of distortion.

With regard to the negative memory effect for one but not the other type of distortion, Heinrich and Schneider (2010) argued that the two types of jitter differed in the spectral spread they caused, and the noise floor was substantially lower for one type of jitter than for the other. Older adults' memory was only affected for the jitter condition in which the signal-to-noise ratio (SNR) was low (i.e., the noise floor was high). Hence, their explanation for the differential effects of the two types of jitter on memory in older adults was that jitter interfered with memory when the spectrum level of the noise floor of the jittered word was high relative to the spectrum level of the word.

The present experiment was designed to investigate an additional factor that might have played a role in explaining the different results for younger and older adults for word distortion, namely, that one type of jittering produced rollover effects in older adults. The following section will review previous findings to rollover and age. At this point, it shall only be noted that several things might have promoted the occurrence of rollover in the older listeners in the study by Heinrich and Schneider (2010): (1) words were distorted, (2) the distorted words were presented at a higher signal level for older than younger adults to ensure equal intelligibility, and (3) rollover can occur at a lower level for older than for younger adults. Together, these factors may have caused subtle effects of rollover on memory for older adults, which were not sufficiently intense to affect intelligibility.

\section{Level Effects and Rollover in Speech Perception}

In the study by Heinrich and Schneider (2010), participants listened to all speech material at $50 \mathrm{~dB}$ above their respective speech threshold (the softest level of speech they could perceive), which is denoted as their sensation level (SL). For younger adults, this translated into an average presentation level of $66 \mathrm{~dB}$ SPL; for older adults, it was a level of $73 \mathrm{~dB}$ SPL. This approach to setting the presentation level had been chosen because older but not younger adults showed signs of presbycusis as measured by audiometric thresholds, even though only older adults with audiometrically normal hearing within the speech range (up to $3 \mathrm{kHz}$ ) had been included in the study. We do not claim that these older listeners had normal hearing with respect to all hearing functions. While one may think that increasing the presentation level in response to increased hearing loss would serve to restore intelligibility, various studies suggest that this is true only to a certain extent. Studies of the effect of presentation level on speech perception accuracy have shown that speech intelligibility increases with increasing presentation level until it reaches an asymptotic value, typically near $100 \%$. Further increases in level not only do not lead to a further increase in intelligibility but also can result in a loss of intelligibility for some listeners. This phenomenon is called the "rollover" effect (Jerger \& Jerger 1971; Dirks et al. 1977; Bess et al. 1979).
Rollover is most often associated with retrocochlear pathology (Martin 1997; Stach 1998) even though a small effect of the magnitude of about $10 \%$ can also be shown for listeners with cochlear dysfunction (Jerger \& Jerger 1971; Dirks et al. 1981), particularly if the audiometric hearing loss is only moderate and does not exceed $45 \mathrm{~dB}$ HL PTA (Jerger et al. 1971). To elicit the effect, the sound level usually needs to exceed 70 dB SPL (Jerger et al. 1971; Studebaker et al. 1999; Miranda \& Pichora-Fuller 2002). Last, the rollover effect appears to increase in prevalence as people age (Jerger \& Jerger 1971; Gang 1976; Dirks et al. 1977; Shirinian \& Arnst 1980).

In fact, in younger normal-hearing adults, a rollover effect can be difficult to elicit, and even be impossible to show, when perceptually intact words in a quiet background are used (Studebaker et al. 1999; Miranda \& Pichora-Fuller 2002). It is more likely to occur in younger adults when words are embedded in background noise (Studebaker et al. 1999) or perceptually distorted (Miranda et al. 2002). Importantly, to elicit the effect, stimulus presentation levels have to be well above the level at which the correct reporting of words reaches its maximum level (Dirks et al. 1977; Studebaker et al. 1999; Miranda \& Pichora-Fuller 2002).

In summary, older listeners are more likely to be affected by rollover than younger adults and are affected at lower intensity levels. Therefore, it is possible that the older listeners in the previous study were approaching or had reached the rollover portion of the speech intelligibility function when tested at 50 $\mathrm{dB}$ SL. It is the goal of the present study to test whether rollover can adversely affect memory even after controlling for intelligibility. As far as we are aware, this is the first study that moves beyond intelligibility as an outcome measure of rollover and examines the effects of high presentation levels on memory performance. We believe that presentation level might adversely affect memory independent of its effects on intelligibility because memory encoding operates on the perceptual representation of a word. If the perceptual representation is compromised due to subtle effects of rollover, possibly even too subtle to manifest themselves in intelligibility, then memory encoding could be compromised as well.

To keep the comparability with Heinrich and Schneider (2010), all speech material was distorted using a technique called jittering (Miranda \& Pichora-Fuller 2002; Pichora-Fuller et al. 2007). This technique also allowed us to equate intelligibility between the two age groups at a level below ceiling while taking into account various degrees of rollover. As a result, conditions with ostensibly more rollover received less jittering to achieve the same intelligibility score as conditions with less rollover. To understand how jittering distorts a sound, consider an undistorted signal, $y(t)$. The jittering process distorts the signal by introducing a variable time delay such that the distorted signal $x(t)$ is given by $y(t-\delta)$, where $\delta$ is a variable that changes randomly over time. Thus, $x(t)$ either leads or lags $y(t)$ depending on whether $\delta$ is positive or negative. The value of $\delta$ is determined by the amplitude of a low pass-filtered white noise. The range of variation of $\delta$ (which has a mean value of 0 ) is determined by the root mean square (rms) value of the low-pass noise. The rate of change in $\delta$ is controlled by the band limit (expressed in $\mathrm{Hz}$ ) of the low-pass noise. For further details concerning this method, see the article by Pichora-Fuller et al. (2007). 
TABLE 1. Details concerning age, gender, years of education, Mill Hill vocabulary score, speech threshold, and stimulus presentation level for younger and older participants for the intelligibility (intell) and memory (mem) tests of Experiments 1 (40 dB SL) and 2 (UCL), respectively. Details for a previous experiment (Heinrich \& Schneider 2010) in which the same stimuli were presented at $50 \mathrm{~dB}$ SL are also provided.

\begin{tabular}{|c|c|c|c|c|c|c|c|c|c|}
\hline \multirow[b]{2}{*}{ Experiment } & \multirow[b]{2}{*}{ Task } & \multirow{2}{*}{$\begin{array}{l}\text { Level } \\
\text { (dB SL) }\end{array}$} & \multirow{2}{*}{$\begin{array}{c}\text { Age Range } \\
\text { (yrs) }\end{array}$} & \multirow{2}{*}{$\begin{array}{l}\text { Age, Mean } \\
\text { (SD) }\end{array}$} & \multirow{2}{*}{$\begin{array}{l}\text { Gender } \\
(\mathrm{M} / \mathrm{F})\end{array}$} & \multirow{2}{*}{$\begin{array}{c}\text { Education (yrs), } \\
\text { Mean (SD) }\end{array}$} & \multirow{2}{*}{$\begin{array}{c}\text { Vocab Score, } \\
\text { Mean (SD) }\end{array}$} & \multicolumn{2}{|c|}{$\begin{array}{l}\text { Left Ear Babble } \\
\text { Threshold (dB SPL) }\end{array}$} \\
\hline & & & & & & & & Mean (SD) & Range \\
\hline Previous study & Intell & 50 & $65-78$ & $67.50(4.48)$ & $5 / 5$ & 13.20 (3.65) & $14.70(1.57)$ & $23.03(4.32)$ & $19-32$ \\
\hline Previous study & Mem & 50 & $19-24$ & $21.40(1.72)$ & $6 / 9$ & $16.40(1.92)$ & 13.67 (1.99) & $15.86(4.54)$ & $11-28$ \\
\hline Previous study & Mem & 50 & $64-79$ & $69.53(4.75)$ & $8 / 7$ & $13.07(2.50)$ & $15.33(2.32)$ & $22.97(4.70)$ & 17-31 \\
\hline 1 & Mem & 40 & $19-23$ & $20.87(1.60)$ & $3 / 12$ & 16.47 (1.69) & 13.40 (1.68) & $14.49(4.49)$ & $8-28$ \\
\hline 1 & Mem & 40 & $65-82$ & $72.73(5.04)$ & $6 / 9$ & $14.53(2.92)$ & $15.80(1.78)$ & $22.57(5.00)$ & $15-32$ \\
\hline 2 & Intell & UCL & $18-24$ & $21.00(2.21)$ & $3 / 7$ & $16.00(1.41)$ & $12.90(1.45)$ & 96.17 (8.39) & $85-110$ \\
\hline 2 & Mem & UCL & $19-25$ & $21.43(1.73)$ & $8 / 15$ & $16.57(2.13)$ & $13.13(1.84)$ & 98.48 (9.39) & $82-110$ \\
\hline
\end{tabular}

Given that we set out to investigate an alternative reason for the memory decrement in one of the jitter conditions in the study by Heinrich and Schneider (2010), we used the same rate of change in time delay as in the condition in question $(100 \mathrm{~Hz}$ bandwidth $[\mathrm{BW}]$ ) and only varied the range of time delays introduced into the signal to bracket the value that yielded an intelligibility score closest to $91 \%$. Moreover, also following the study by Heinrich and Schneider (2010), we used a combined baseline (words presented in quiet with babble optionally present between the word pairs) to increase power in the statistical analyses performed.

\section{EXPERIMENT 1}

Heinrich and Schneider (2010) presented jittered word pairs at an SL of $50 \mathrm{~dB}$. In this experiment, the jittered words were presented at $40 \mathrm{~dB}$ SL. Because the translation of SPL to SL varies between listeners and because different groups of participants were used in the two studies, a reduction of presentation level from 50 to $40 \mathrm{~dB}$ SL resulted in a change of average SPL from $66 \mathrm{~dB}$ to $55 \mathrm{~dB}$ for younger adults and from $73 \mathrm{~dB}$ to $65 \mathrm{~dB}$ for older adults. Because the different groups used for the 40 and $50 \mathrm{~dB}$ SL conditions had different average audiometric sensitivity, a $10 \mathrm{~dB}$ reduction in SL did not result in an equivalent reduction in average SPL. On the basis of rollover studies, we expected the higher average presentation level of $66 \mathrm{~dB}$ SPL in younger listeners to be well below the point at which rollover occurs in younger adults, hence lowering the presentation level to $55 \mathrm{~dB}$ SPL was not expected to improve the perceptual representation of the stimulus and therefore memory. Conversely, for older adults, the picture was less clear. Possibly, $73 \mathrm{~dB}$ SPL (50 dB SL) in the previous study represented the lower end of the rollover region, and therefore lowering the level to $65 \mathrm{~dB}$ SPL ( $40 \mathrm{~dB}$ SL) might eliminate subtle negative effects on the perceptual representation of the word pairs and improve memory.

\section{Methods}

The stimuli, apparatus, testing protocols, subject pools, and research technicians were identical to earlier studies (Murphy et al. 2000; Heinrich et al. 2008; Heinrich \& Schneider 2010).
Hence, we feel justified to use comparison data where applicable.

\section{Participants}

Fifteen younger and 15 older adults participated in the main portion of this study. An additional 10 younger and 9 older adults participated in a preliminary experiment to determine the degree of jitter to be employed in the main experiment. The details concerning age, years of education, and Mill Hill vocabulary score of both age groups in Experiments 1 and 2 are given in Table 1. The details of the $50 \mathrm{~dB}$ SL comparison groups taken from the study by Heinrich and Schneider (2010) are also reprinted in Table 1. Younger adults were undergraduate students at the University of Toronto. Older participants were adults from the local community. In exchange for their participation, participants received $\$ 10$ per hour.

\section{Material}

The same speech material as in previous studies (Heinrich et al. 2008; Heinrich \& Schneider 2010) was used. The material consisted of a total of 400 two-syllable common nouns with a frequency of more than 1 per million (Kucera \& Francis 1967). The individual words, spoken by a female speaker, were digitally recorded at a sampling rate of $20 \mathrm{kHz}$ within a single-walled sound-attenuating chamber. All recordings had similar rms values. For stimulus presentation, the words were delivered through a 16-bit digital-to-analog converter (TDT DD1) followed by a 10-kHz low-pass filter (TDT FT6-2, $60 \mathrm{~dB}$ attenuation at $11.5 \mathrm{kHz}$ ), a programmable attenuator (TDT PA4), and a weighted signal mixer (TDT SM3). All testing took place in a double-walled sound-attenuating chamber. For a more detailed description of the material, see the article by Heinrich and Schneider (2010).

\section{Procedures}

Audiometric Testing • Pure-tone air conduction thresholds were determined for all participants for nine frequencies between 0.25 and $8 \mathrm{kHz}$ and used as inclusion criteria for the study. This was done to minimize the possibility that audiometric differences could act as an explanation for the potential differences in the memory task between the two age groups. First the left then the right ear was tested using an Interacoustics Model AC5 audiometer. To participate in the study, participants were required to have pure-tone air conduction 


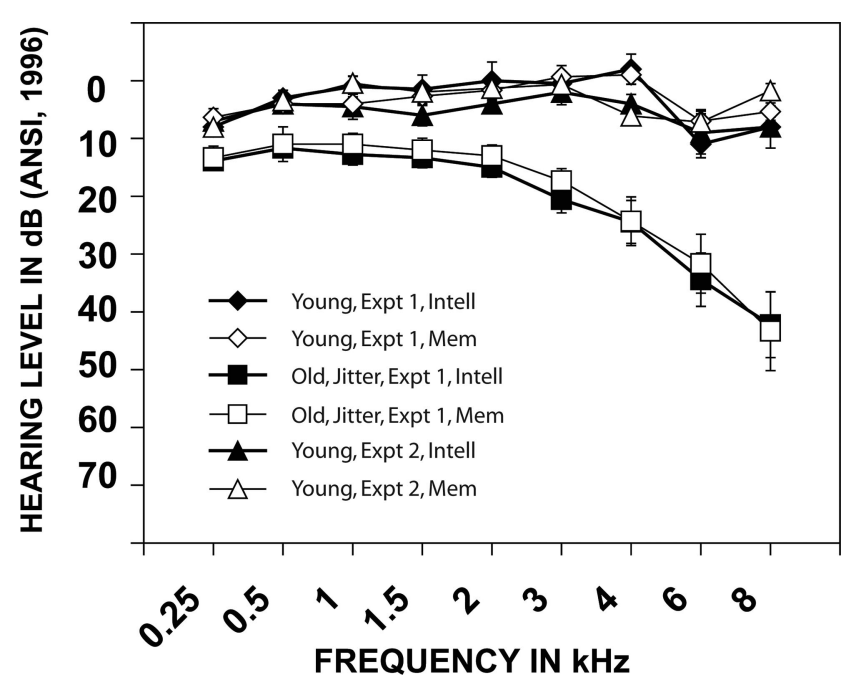

Fig. 1. Average audiometric thresholds and SE as a function of test frequency for all participants in the intelligibility and memory parts of experiments 1 and 2. A hearing level of $0 \mathrm{~dB}$ represents the expected performance for normal-hearing younger adults (ANSI 1996).

thresholds of $\leq 30 \mathrm{~dB}$ HL (ANSI 1996) at all frequencies between 0.25 and $2 \mathrm{kHz}, \leq 35 \mathrm{~dB} H \mathrm{HL}$ at $3 \mathrm{kHz}$ in the test ear, and $\leq 45 \mathrm{~dB} \mathrm{HL}$ at $4 \mathrm{kHz}$. There were no requirements for frequencies $>4 \mathrm{kHz}$. Moreover, listeners were permitted to exceed these criteria at one test frequency by $5 \mathrm{~dB}$ HL. Figure 1 shows average audiometric thresholds for the left ear of younger and older participants in this and the next experiment. The right ear (not shown) showed comparable results. Young listeners generally showed very little hearing loss in either ear across all tested frequencies. Older listeners, as a group, showed a symmetrical and sloping hearing loss in both ears that progressed from a mild loss in frequencies below $3 \mathrm{kHz}$ to a moderate loss in the frequencies between 3 and $8 \mathrm{kHz}$. The mean audiograms looked very similar for the groups participating in the perceptual and memory parts of the two experiments.

Babble Threshold - The procedure was the same as in previous studies (Murphy et al. 2000; Heinrich et al. 2008; Heinrich \& Schneider 2010). To briefly summarize, the threshold for detecting a speech babble was determined for each individual listeners by means of an adaptive two-interval forced-choice procedure. A 12-talker babble segment was presented in one of two randomly chosen intervals, and the remaining interval contained silence. The babble was taken from the modified Speech Perception in Noise (SPIN) test
(Bilger et al. 1984). After initiating each trial by pressing the middle button on a three-button box, the listener indicated which of two 1.5-sec long intervals contained the babble. Lights above the two outer buttons indicated when an interval occurred, and the light associated with the interval containing the babble flashed immediately after the button press for feedback. We used a three-down, one-up adaptive technique (79\%, Levitt 1971) to determine the babble threshold starting at $50 \mathrm{~dB}$ SPL. The session was terminated after 12 reversals (change from decreasing to increasing intensity level or vice versa) in total, but only the recordings from the last eight were averaged for the babble threshold. The speech threshold was obtained for the right ear first and the left ear second. Because stimuli were presented to the left ear during these experiments, Table 1 presents babble thresholds for the left ear only.

Finding the Appropriate Jitter Levels • The 400 words used in the memory part of the experiment were presented in 10 lists of 40 words each. The words in each list were jittered at one of five rms values $(0.3,0.35,0.4,0.45$, and $0.5 \mathrm{msecs})$, which had been selected on the basis of pilot testing to bracket a word report score of $91 \%$. The rate of change given by the bandwidth of the noise signal was kept constant at $100 \mathrm{~Hz}$. The pairing of lists with rms values, as well as the order of list presentation, was counterbalanced across participants. The words in a list were presented over earphones (TDH-49) to the left ear at a rate of one every 5 secs at an intensity level of $40 \mathrm{~dB}$ SL, that is, $40 \mathrm{~dB}$ above each individual's babble threshold (SL).

Ten younger and nine older participants were asked to listen to and repeat each word. They were encouraged to guess whenever they did not hear a word properly. There were no practice trials, and no feedback was given. Participants were given a short break after the presentation of the fifth list. The experimenter, positioned outside the sound-attenuating booth, monitored the participant's performance by means of headphones. The listener's answers were compared with a checklist containing all the words, and misidentified words were noted down. Moreover, all answers were tape-recorded and doublechecked. For that purpose, an individual different from the experimenter listened to the tape and compared the participant's answer with the answer written down by the experimenter. Discrepancies, which were very rare, were settled by consensus. Table 2 details average word report scores for each jitter level in this and the following experiment. The table also displays the word report scores for jittered words presented at the same five distortion levels but at $50 \mathrm{~dB}$ SL in the previous study by Heinrich and Schneider (2010). When the words were presented at $40 \mathrm{~dB}$ SL, the rms level that yielded an intelligi-

TABLE 2. Mean intelligibility scores (and SE) for younger and older adults for each rms distortion level tested in Experiments 1 (40 dB $\mathrm{SL}$ ) and 2 (UCL). The results of a previous experiment (Heinrich \& Schneider 2010) in which the same words were presented at $50 \mathrm{~dB}$ SL are also provided.

\begin{tabular}{|c|c|c|c|c|c|c|c|c|}
\hline Experiment & Age Group & $\mathrm{N}$ & Level (dB SL) & \multicolumn{5}{|c|}{ Perceptual Accuracy (in \%) per rms Level (msecs) } \\
\hline Previous experiment & Young & 10 & 50 & $95.88(0.83)$ & $95.25(0.69)$ & $95.63(0.68)$ & $92.75(1.03)$ & $90.25(2.33)$ \\
\hline \multirow[t]{2}{*}{1} & Young & 10 & 40 & $92.88(2.15)$ & $91.63(1.95)$ & $91.00(1.62)$ & $86.75(2.75)$ & $84.13(2.43)$ \\
\hline & Old & 9 & 40 & $93.89(1.13)$ & $91.39(1.62)$ & $90.56(1.27)$ & $87.78(1.10)$ & $83.33(2.86)$ \\
\hline 2 & Young & 10 & UCL & $92.63(1.81)$ & $93.13(1.57)$ & $92.63(1.13)$ & 90.50 (1.59) & $88.13(2.22)$ \\
\hline
\end{tabular}




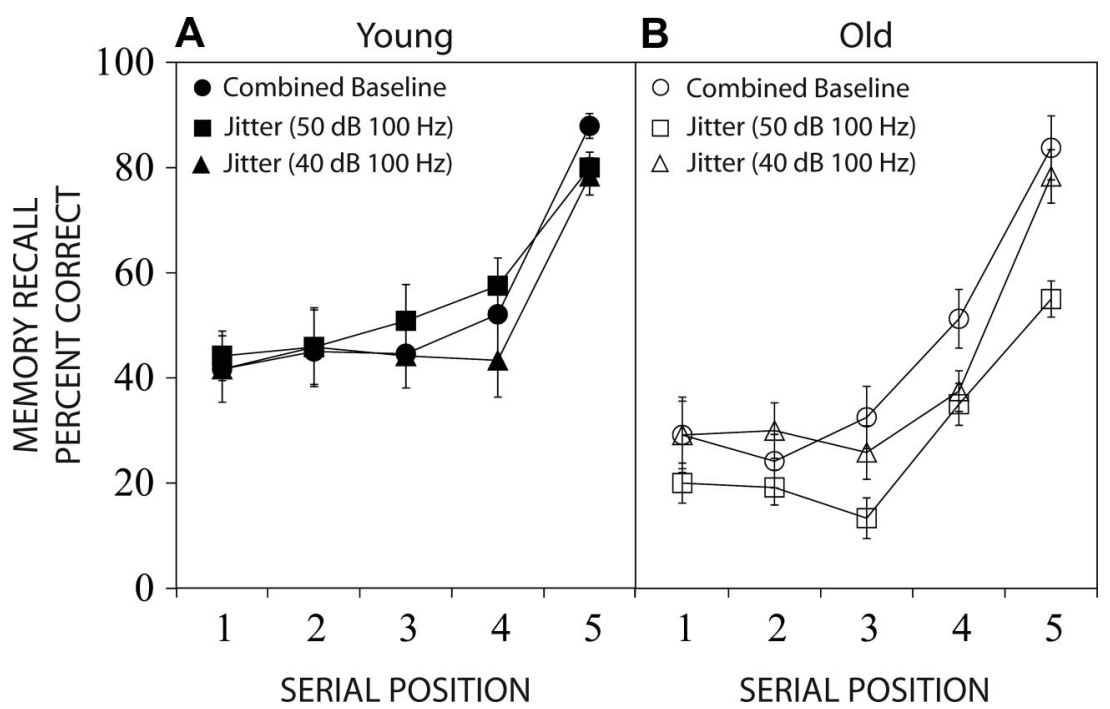

Fig. 2. Average percentage of correctly recalled word pairs and SE of the mean for word pairs presented in jitter (100 Hz bandwidth) at 50 and $40 \mathrm{~dB}$ SL and for an unjittered baseline. Results for younger and older listeners are represented in separate panels. The results for jittered stimuli presented at $50 \mathrm{~dB} S \mathrm{~L}$ and the unjittered baseline are reprinted from Heinrich and Schneider (2010).

bility performance closest to the $91 \%$ was 0.4 msecs for younger and $0.35 \mathrm{msecs}$ for older listeners. These two rms values were subsequently used to distort the words for the memory task. This procedure assumes that the intelligibility results obtained for the groups of younger and older adults in this part of the experiment will generalize to a similar degree to the respective age group in the next part of the study. The comparable size of the standard error in Table 2 between both groups suggests that this assumption is reasonable.

Word Recall • Fifteen participants in each age group listened to 40 lists containing five word pairs each. The words were randomly paired, with any obvious association between two words being avoided. Four seconds after a short warning tone (1 $\mathrm{kHz}$ at $90 \mathrm{~dB}$ SPL for 500 msecs) had indicated the beginning of a list, the first word of the first pair was presented. Within each pair, there was a silent period of 100 msecs between the words; the word pairs were played with a presentation rate of one pair every 4 secs. Four seconds after the start of the final word pair, a short warning tone indicated the beginning of the recall phase. After another 4 secs, participants were cued with the first word from one of the five previously presented word pairs and were asked to recall the second word of the pair. Only one pair from each list was cued. There was no time limit placed on the recall. Participants were encouraged to guess.

The first serial position refers to the first word pair and so forth. List order was identical for all participants, and the serial position of each word pair within the five word pair list was tested an equal number of times (eight times) within a session. The order in which the serial positions were tested was independently and randomly determined for each participant. There were no practice trials, and no feedback was provided. There was a break after the presentation of the first 20 lists. Memory performance for each serial position was measured. At encoding, all words were jittered according to the levels in Table 2. When one of the words was re-presented at the retrieval, this word was undistorted.

\section{Results}

Figure 2 plots memory performance (percentage of correctly recalled words) as a function of serial position for younger (plot A) and older (plot B) adults (triangle). For comparison purposes, two results from a previous study (Heinrich \& Schneider 2010) are also displayed: (1) a combined baseline for memory of undistorted words (circle), (2) memory performance for jittered words presented at $50 \mathrm{~dB}$ SL for which the rate of change (signified by bandwidth) was identical to the current experiment but whose rms distortion values were adjusted to slightly higher value $(0.5 \mathrm{msecs}$ for younger and 0.4 msecs for older adults) to yield 91\% perceptual accuracy (square).

The result of the present study was compared with these earlier results in an Age (Young, Old) by Serial Position by Experiment (combined baseline, $40 \mathrm{~dB}$ SL $100 \mathrm{~Hz}, 50 \mathrm{~dB}$ SL $100 \mathrm{~Hz}$ ) mixed-measures ANOVA, with Age and Experiment as between factors and Serial Position as within-subject factor. The analysis shows main effects of Serial Position $(F[4,456]=$ 140.56, MSE $=259.00$, partial $\left.\eta^{2}=0.55, p<0.0001\right)$ and Age $\left(F[1,114]=28.097, \mathrm{MSE}=1208.75\right.$, partial $\eta^{2}=0.20$, $p<0.0001)$. In addition, all two-way interactions were significant: Age by Serial Position $(F[4,456]=3.98$, MSE $=$ 259.00, partial $\left.\eta^{2}=0.034, p=0.003\right)$; Serial Position by Experiment $\left(F[8,456]=2.76\right.$, MSE $=259.00$, partial $\eta^{2}=$ $0.05, p=0.006)$; and Age by Experiment $(F[2,114]=3.28$, $\mathrm{MSE}=1208.75$, partial $\left.\eta^{2}=0.05, p=0.041\right)$.

As can be seen in Figure 2, there is no effect of Experiment and no Experiment by Serial Position interaction for younger adults. There is only the usual main effect of serial position $\left(F[4,228]=56.44, \mathrm{MSE}=252.60\right.$, partial $\eta^{2}=0.50, p<$ $0.0001)$. Hence, for younger adults, there is only a serial position effect while the presentation level of the jittered words has no significant effect on memory. For older adults, in addition to main effects of Serial Position $(F[4,228]=87.33$, MSE $=265.40$, partial $\eta^{2}=0.61, p<0.0001$, GreenhouseGreiser correction for violation of sphericity) and Experiment 
$\left(F[2,57]=6.20, \mathrm{MSE}=975.51\right.$, partial $\left.\eta^{2}=0.18, p=0.004\right)$, there is also a significant Serial Position by Experiment interaction $\left(F[8,228]=2.40\right.$, MSE $=265.40$, partial $\eta^{2}=$ $0.08, p=0.024$, Greenhouse-Greiser correction for violation of sphericity). Further testing revealed that the interaction effect involving Experiment in older adults occurs because at serial position 2 for the baseline condition and at serial position 4 for the $40 \mathrm{~dB}$ SL $100 \mathrm{~Hz}$ jitter condition, recall drops to levels close to the $50 \mathrm{~dB}$ SL $100 \mathrm{~Hz}$ jitter condition. When these serial positions are removed from the model, the interaction disappears. For the remaining three serial positions, there is a clear drop in memory for jittered words presented at $50 \mathrm{~dB}$ SL. This result, presented in Figure 2, suggests that a high presentation level can impair memory, a result that is consistent with and extends the existence of rollover in older listeners.

Additional evidence for the fact that older but not younger listeners were indeed at the cusp of the rollover effect at $50 \mathrm{~dB}$ $\mathrm{SL}$ is provided by the function that relates intelligibility to distortion. When we analyzed the intelligibility values for each distortion level $(0.3,0.35,0.4,0.45$, and 0.5$)$ as within-subject factor and two presentation levels (40 dB SL $100 \mathrm{~Hz}$ and $50 \mathrm{~dB}$ SL $100 \mathrm{~Hz}$ ) as between-subject factor in a mixed-measures ANOVA for younger listeners, there was a main effect of distortion level $\left(F[4,72]=11.62\right.$, MSE $=15.885$, partial $\eta^{2}=$ $0.39, p<0.001)$ and of presentation level $(F[1,18]=5.48$, MSE $=99.80$, partial $\eta^{2}=0.23, p=0.03,50 \mathrm{~dB}$ SL $>40 \mathrm{~dB}$ $\mathrm{SL})$ but no interaction $(F<1)$. When the same analysis was run for older listeners, there was only a main effect of distortion level $\left(F[4,68]=15.38, \mathrm{MSE}=15.43\right.$, partial $\eta^{2}=0.48, p<$ 0.001 ) (all other $F$ 's $<1$ ). Hence, reducing the presentation level from 50 to $40 \mathrm{~dB}$ SL significantly reduced the level of distortion required for $91 \%$ intelligibility in younger adults. In other words, younger adults could tolerate more distortion at the higher presentation level for a set level of intelligibility. The same was not true for older adults. If one can assume that intelligibility is a U-shaped function that improves and becomes more resilient against distortion with increasing sound level, and then reaches a peak and starts to decline again at high levels, it looks as if younger listeners had not yet reached that peak at $40 \mathrm{~dB}$ SL whereas older adults had. This in turn makes it more likely that some older listeners may have been at the verge of crossing over to the rollover portion of the function. Interestingly, the effect of high presentation level was not pronounced enough to impair intelligibility, yet an adverse effect occurred for older adults' memory performance. Because this memory effect in older adults was associated with an average presentation level of $73 \mathrm{~dB}$ SPL, we might expect to find additional negative effects on memory at presentation levels higher than $73 \mathrm{~dB}$ SPL in older adults when words are distorted.

\section{EXPERIMENT 2}

Experiment 2 aimed to replicate for younger adults the adverse effect that high presentation level can have on memory performance of older adults. We chose a presentation level at which some rollover is likely to occur, even for younger listeners. Our choice of presentation level was informed by a study by Miranda and Pichora-Fuller (2002) who presented younger adults with jittered words in an intelligibility task at different presentation levels. At a sound level near the uncom- fortable listening level (UCL) (Stach 1998), jittered words showed a clear rollover effect. Therefore, we chose the UCL as our presentation level. According to Mueller and Bright (1994), the UCL is a level somewhere between the initial discomfort experienced when first listening to a loud sound and extreme and sustained discomfort that arises with very loud sounds.

\section{Methods \\ Participants}

A total of 33 younger adults participated in the study. The details concerning age, years of education, and Mill Hill vocabulary score are given in Table 1 . All participants were undergraduate students at the University of Toronto and received \$10 per hour in exchange for their participation.

\section{Material}

The same speech material as in Experiment 1 was used.

\section{Procedures}

Audiometric Testing - Audiometric testing procedures were the same as in experiment 1 . The results for this second group of younger listeners are displayed in Figure 1.

Finding the Uncomfortably Loud Listening Level • Instead of obtaining the participants' speech threshold, their uncomfortable listening level (UCL) was determined following the procedure proposed by Mueller and Bright (1994). A continuous speech file was presented to the participants' right ear at a level of $60 \mathrm{~dB}$ SPL. After listening to the speech stimulus for some time, the participant rated its loudness on a 6-point scale containing the following categories: soft, slightly soft, comfortable, slightly loud, annoying, and uncomfortably loud. Then, the level of the speech signal was increased by 5 $\mathrm{dB}$, and the rating was repeated. The intensity level of the stimulus was increased until the listener reported the stimulus to be uncomfortably loud or until a presentation level of $110 \mathrm{~dB}$ SPL was reached. The procedure was then repeated in the same ear with starting levels of 70 and $50 \mathrm{~dB}$ SPL, respectively, to minimize the influence of anchor effects on ratings. Subsequently, the left ear was tested with the same protocol. The three UCL measurements obtained for each ear were averaged, and the presentation level of the word pairs was set to $5 \mathrm{~dB}$ below this average or to $100 \mathrm{~dB}$ SPL if the averaged UCL was higher than $105 \mathrm{~dB}$ SPL. The mean intensity levels for uncomfortable listening in this experiment are displayed in Table 1 under the heading "babble threshold." UCLs did not differ significantly between those listeners in the perception and the memory part of the study.

Finding the Appropriate Jitter Levels - After the uncomfortable listening level had been found for each listener, intelligibility for single words was measured for various amounts of jitter. The same procedure as in Experiment 1 was used. A group of 10 listeners was tested at each of five jitter levels $(0.3,0.35,0.4,0.45$, and 0.5 msecs $)$ at their individual UCL. The results are summarized in Table 2. They show that an rms value of 0.45 msecs produced an intelligibility score closest to $91 \%$. A mixed-measures ANOVA with distortion level as within-subject variable and presentation level (50 dB SL $100 \mathrm{~Hz}$, UCL $100 \mathrm{~Hz}$ ) as between-subject variable only showed a reliable effect of jitter level $(F[4,72]=7.47, \mathrm{MSE}=$ $\left.13.49, \eta^{2}=0.29, p<0.001\right)$ but not of presentation level or the interaction between the two. 


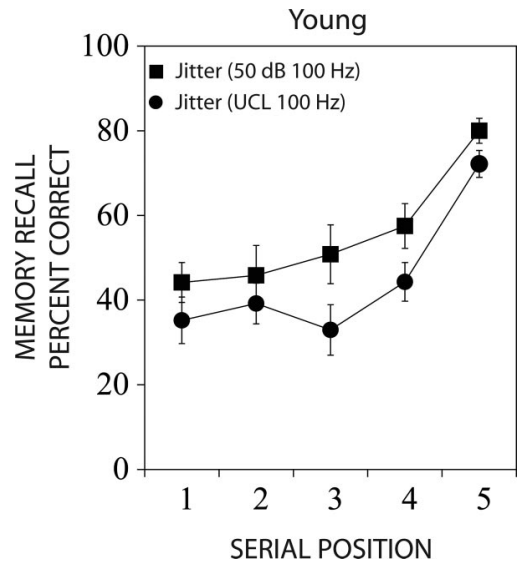

Fig. 3. Average percentage of correctly recalled word pairs and SE of the mean by younger listeners for word pairs presented jittered at $100 \mathrm{~Hz}$ bandwidth and presented at $50 \mathrm{~dB} S \mathrm{SL}$ and uncomfortable listening level. The results for the $50 \mathrm{~dB} S \mathrm{~L}$ condition are reprinted from Heinrich and Schneider (2010).

Word Recall • The word pairs were jittered at an rms level of 0.45 msecs and a bandwidth of $100 \mathrm{~Hz}$ and presented at the individual UCL to each of the 23 younger participants. The procedure of the memory test itself was the same as in Experiment 1.

\section{Results}

Figure 3 plots the percentage of correctly recalled words as a function of serial position in this experiment (circle). For comparison purposes, memory performance for jittered words presented at $50 \mathrm{~dB}$ SL is replotted from Heinrich and Schneider (2010) (square). In that study, the rate of change (signified by bandwidth) for the jitter was identical with that used in the current experiment, but the rms value was adjusted to a slightly higher value $(0.5 \mathrm{msecs})$ to yield $91 \%$ perceptual accuracy.

The result of the present study was compared with the earlier result in a Serial Position by Presentation Level mixedmeasures ANOVA with Presentation Level (50 dB SL $100 \mathrm{~Hz}$, UCL $100 \mathrm{~Hz}$ ) as between and Serial Position as within-subject factor. The analysis shows main effects of Serial Position $\left(F[4,144]=26.17, \mathrm{MSE}=293.07\right.$, partial $\eta^{2}=0.42, p<$ $0.0001)$ and Presentation Level $(F[1,36]=4.09$, MSE $=$ 1341.62, partial $\left.\eta^{2}=0.10, p=0.05\right)$. The interaction was not significant $(F<1)$.

The results of Experiment 2 for younger adults show the same effect of level as the results of Experiment 1 for older adults. First, there was no significant increase in intelligibility when the presentation level was increased from $50 \mathrm{~dB}$ SL to the UCL. This suggests that younger listeners had reached at least the asymptote on the performance-intensity function for jittered words and that perceptual accuracy was starting to decrease with further increases in level. Second, even though perceptual accuracy was equated between $50 \mathrm{~dB}$ SL and UCLs, memory performance was still lower for the UCL condition than for the $50 \mathrm{~dB}$ SL condition.

\section{Discussion}

The study investigated the effect of high presentation level on memory performance for younger and older listeners. The study was inspired by work on the rollover effect, which showed that high presentation levels can lead to decreased intelligibility and that older adults are affected more often and at lower levels. The results show that high presentation levels for distorted words adversely affect memory, even after equating for word intelligibility. This suggests that a high presentation level exerts a unique effect on memory performance that is not accounted for by intelligibility. The study also shows that both age groups are affected but that older adults are vulnerable to this effect at lower presentation levels than younger adults. We are aware that older adults as a group are less homogeneous than younger adults and that age-related hearing loss adds internal noise for older listeners. However, by choosing a lower jitter level for older listeners, we hoped to equate for these differences in peripheral functioning.

There still remains the question of why presenting distorted words at a sensation level of $50 \mathrm{~dB}$ SL resulted in memory loss for one of the jitter conditions but not the other. Recall that from a spectral point of view, the level of spectral splatter produced by one kind of jitter (the $100 \mathrm{~Hz}$ bandwidth studied here) was much higher than that produced by words jittered with a $500 \mathrm{~Hz}$ bandwidth, with memory being affected for a presentation level of $50 \mathrm{~dB}$ SL in the former but not in the latter. Because rollover is more likely to occur in a noise background (Studebaker et al. 1999), we attributed the difference in the amount of memory rollover to the differences in spectrum levels of the spectral splatter in the jittered words.

It would also be important to determine the extent of any "memory" rollover effect in listeners with a clinically significant hearing loss. Because the rollover effect is likely to occur at lower levels in such individuals (Martin 1997; Stach 1998) and because the compression algorithms employed in hearing aids inevitably introduce distortion, we might expect such individuals to experience a significant memory impairment (as measured in a serial position experiment) at presentation levels that are lower than those of normal-hearing listeners. Information about potential memory impairments due to high levels of amplification and/or distortion could then be used to inform the clinician when fitting hearing aids.

This study also demonstrates the importance of choosing an appropriate presentation level, especially when testing older adults. Louder is not always better, not only with respect to speech recognition but also with respect to memory for heard material. Moreover, the results suggest that there might be situations in which memory performance might serve as a sensitive measure of perceptual manipulations, particularly when perceptual factors may exert a strong effect on the processes subsequent to intelligibility.

\section{ACKNOWLEDGMENTS}

The authors thank Jane Carey and Neda and Alham Chelehmalzadeh for their assistance in data collection.

This work was supported by grants from the Natural Sciences and Engineering Research Council of Canada (RGPIN 9952) and Canadian Institutes of Health Research (MOP 15359) and also supported, in part, by a Canadian Institutes of Health Research Training grant (STP 53875) and the International Council for Canadian Studies (to A. H.).

Address for correspondence: Antje Heinrich, MRC Institute for Hearing Research, University Park, Science Road, Nottingham, NG7 2 RD, United Kingdom. E-mail: antje.heinrich@ihr.mrc.ac.uk.

Received August 16, 2010; accepted November 29, 2010. 


\section{REFERENCES}

Alain, C., \& McDonald, K. L. (2007). Age-related differences in neuromagnetic brain activity underlying concurrent sound perception. $J \mathrm{Neu}-$ rosci, 27, 1308-1314.

ANSI. (1996). American National Standard Specification for Audiometers S3.6-1996. New York, NY: American National Standards Institute.

Bess, F. H., Josey, A. F., Humes, L. E. (1979). Performance intensity functions in cochlear and eighth nerve disorders. Am J Otol, 1, 27-31.

Bilger, R. B., Nuetzel, M. J., Rabinowitz, W. M., et al. (1984). Standardization of a test of speech perception in noise. J Speech Hear Res, 27, $32-48$.

Bregman, A. S. (1990). Auditory Scene Analysis: The Perceptual Organization of Sound. Cambridge, MA: MIT Press.

Brown, J. (1958). Some tests of the decay theory of immediate memory. Q J Exp Psychol, 10, 12-21.

Carpenter, P. A., \& Just, M. A. (1989). The Role of Working Memory in Language Comprehension. In D. Klahr \& K. Kotovsky (Eds). Complex Information Processing: The Impact of Herbert A. Simon (pp. 31-68). Hillsdale, NJ: Erlbaum.

Craik, F. I. M. (1983). On the transfer of information from temporary to permanent memory. Philos Trans R Soc Lond B Biol Sci, 302, 341-359.

Craik, F. I. M. (2002). Levels of processing: Past, present, and future? Memory, 10, 305-318.

Craik, F. I. M., \& Lockhart, R. S. (1972). Levels of processing: A framework for memory research. J Verb Learn Verb Behav, 11, 671-684.

Dirks, D. D., Kamm, C. A., Bower, D., et al. (1977). Use of performanceintensity functions for diagnosis. J Speech Hear Disord, 42, 408-415.

Dirks, D. D., Kamm, C. A., Dubno, J. R., et al. (1981). Speech recognition performance at loudness discomfort level. Scand Audiol, 10, 239-246.

Floden, D., Stuss, D. T., Craik, F. I. M. (2000). Age differences in performance on two versions of the Brown-Peterson task. Neuropsychol Dev Cogn B Aging Neuropsychol Cogn, 7, 245-259.

Gang, R. P. (1976). The effects of age on the diagnostic utility of the rollover phenomenon. $J$ Speech Hear Disord, 31, 63-69.

Hebb, D. O. (1949). Organization of Behaviour. New York, NY: Wiley.

Heinrich, A., \& Schneider, B. A. (2010). Elucidating the effects of ageing on remembering perceptually distorted word pairs. $Q J$ Exp Psychol, $1-20$.

Heinrich, A., Schneider, B. A., Craik, F. I. M. (2008). Investigating the influence of continuous babble on auditory short-term memory performance. Q J Exp Psychol, 61, 735-751.

Jerger, J., \& Jerger, S. (1971). Diagnostic significance of PB word functions. Arch Otolaryngol, 93, 573-580.

Kahneman, D. (1973). Attention and Effort. Englewood Cliffs, NJ: Prentice-Hall.

Kucera, H., \& Francis, W. N. (1967). Computational Analysis of PresentDay American English. Providence, RI: Brown University Press.

Levitt, H. (1971). Transformed up-down methods in psychoacoustics. $J$ Acoust Soc Am, 49, 467-477.
Madigan, S. A., \& McCabe, L. (1971). Perfect recall and total forgettingProblems for models of short-term memory. J Verb Learn Verb Behav, 10, 101-106.

Martin, F. N. (1997). Introduction to Audiology (6th ed.). Boston, MA: Allyn and Bacon.

Miranda, T. T., \& Pichora-Fuller, M. K. (2002). Temporally jittered speech produces performance intensity, phonetically balanced rollover in young normal-hearing listeners. $J$ Am Acad Audiol, 13, 50-58.

Mueller, H. G., \& Bright, K. E. (1994). Selection and Verification of Maximum Output. In M. Valente (Ed). Strategies for Selecting and Verifying Hearing Aid Fittings (pp. 38-63). New York, NY: Thieme Medical Publishers.

Murphy, D. R., Craik, F. I. M., Li, K. Z. H., et al. (2000). Comparing the effects of aging and background noise on short-term memory performance. Psychol Aging, 15, 323-334.

Peterson, L. R., \& Peterson, M. J. (1959). Short-term retention of individual verbal items. J Exp Psychol, 58, 193-198.

Pichora-Fuller, M. K., \& Singh, G. (2006). Effects of age on auditory and cognitive processing: Implications for hearing aid fitting and audiologic rehabilitation. Trends Amplif, 10, 29-59.

Pichora-Fuller, M. K., Schneider, B. A., Daneman, M. (1995). How young and old adults listen to and remember speech in noise. J Acoust Soc Am, 97, 593-608.

Pichora-Fuller, M. K., Schneider, B. A., MacDonald, E., et al. (2007). Temporal jitter disrupts speech intelligibility: A simulation of auditory aging. Hear Res, 223, 114-121.

Rabbitt, P. M. A. (1968). Channel-capacity intelligibility and immediate memory. Q J Exp Psychol, 20, 241-248.

Ronnberg, J., Rudner, M., Foo, C., et al. (2008). Cognition counts: A working memory system for ease of language understanding (ELU). Int J Audiol, 47, S99-S105.

Sarampalis, A., Kalluri, S., Edwards, B., et al. (2009). Objective measures of listening effort: Effects of background noise and noise reduction. $J$ Speech Lang Hear Res, 52, 1230-1240.

Schneider, B. A., Daneman, M., Murphy, D. R., et al. (2000). Listening to discourse in distracting settings: The effects of aging. Psychol Aging, $15,110-125$.

Shirinian, M. J., \& Arnst, D. J. (1980). PI-PB rollover in a group of aged listeners. Ear Hear, 1, 50-53.

Snyder, J. S., \& Alain, C. (2005). Age-related changes in neural activity associated with concurrent vowel segregation. Brain Res Cogn Brain Res, 24, 492-499.

Stach, B. A. (1998). Clinical Audiology-An Introduction. San Diego, CA: Singular Publishing Group.

Studebaker, G. A., Sherbecoe, R. L., McDaniel, D. M., et al. (1999). Monosyllabic word recognition at higher-than-normal speech and noise levels. J Acoust Soc Am, 105, 2431-2444.

Warren, R. M. (1970). Perceptual restoration of missing speech sounds. Science, 167, 392-393. 